\title{
Vortex simulation of active control strategies for transitional backward-facing step flows
}

\author{
Emmanuel Creuséa ${ }^{a}$, André Giovannini ${ }^{\mathrm{b}}$, Iraj Mortazavi c,* \\ ${ }^{a}$ LAMAV, Université de Valenciennes, 59313 Valenciennes Cedex 09, France \\ Equipe SIMPAF, INRIA Futurs, site de Lille \\ ${ }^{\mathrm{b}}$ IMFT, 118, route de Narbonne, 31062 Toulouse, France \\ ${ }^{\mathrm{c}}$ Université de Bordeaux, IMB, INRIA Futurs, Projet MC2 \\ 33405 Talence Cedex, France
}

\begin{abstract}
In this work a vortex method is used to simulate an incompressible two-dimensional transitional flow over a backward-facing step. The simulations are validated for two different Reynolds numbers comparing to previous studies. Then, two different control strategies are implemented to modify the shedding, the recirculation zone behind the step and the transport in the channel. The first technique consists in using a pulsing inlet velocity and the second one is based on local oscillating jets implemented on the step vertical wall. The influence of these controls on several characteristic functionals related to the flow is carefuly investigated. Both, openloop and closed-loop active control approaches are performed in order to choose the most efficient control methods.
\end{abstract}

Key words: Vortex method, Active control, Backward-facing step

\section{Introduction}

Control of internal separated and reattaching flows is motivated by a wide amount of engineering applications, like sudden changes of sections or corners in pipes, cavitation in pumps, mixing in diffusers or combustors. Targets to reach are very diversified, according to the wanted effect: Drag reduction, noise suppression, mixing enhancement, flow stabilization, etc...

\footnotetext{
* Corresponding author

Email address: mortaz@math.u-bordeaux1.fr (Iraj Mortazavi ).
} 
In this kind of flow, the backward-facing step test is one of the most studied cases, because this problem exhibits all the essential features of internal separated flows. Indeed, the pressure increases in the direction of the flow, which causes the boundary layer to separate from the solid surface. The flow reattaches downstream, forming a recirculation bubble. In the same time, this configuration allows some important simplifications with respect to more complex cases. for example, the separation point is a priori known to be located at the edge of the step.

Bibliographic data about the backward-facing step is incredibly wide. Nevertheless, some references among the available papers are already useful to have a large overview. Description of such a flow was given on several dimensioned geometries for laminar, transitional or turbulent regimes. Armaly et al [1] investigated the detailed description from the laminar to the turbulent regime $(70<R e<8000)$, both experimentally and numerically. Their study focused not only on the primary zone of recirculation attached to the backward-facing step, but also on the additional regions of recirculation. They confirmed that available computer codes for flow predictions can be successfully employed to compute backward-facing step flows. Le et al [18] worked on the pressure fluctuation contours and on the reattachment length obtained by a direct numerical simulation for a turbulent regime, and were interested in the skin friction coefficient particularly high in the recirculation regions. Another reference on the skin friction distribution is the paper of Spazzini et al [27], which analysed in details the behavior of instantaneous wall shear stresses downstream of the step. More recently, Wee et al [29] and Yi et al [31] considered a two-dimensional direct numerical simulation to obtain the dominant absolute mode frequency and to investigate large eddy formation and shedding phenomena in the backward-facing step flow as well as instabilities which occur.

Papers on control of such flows are also available. Chun and Sung [10] experimentally produced excitations to separated flow by means of a sinusoidally oscillating jet issuing from a thin slit near the separation line for a turbulent regime. The effects of the amplitude and of the frequency on the reattachment length were analysed. More recently, Kaiktsis anf Monkewitz [16] investigated the global destabilization of a two-dimensional flow over a backward-facing step embedded in a channel using self-excited oscillations of the entire flow, induced by an appropriate local blowing and suction device. Wengle et al [28] experimentally and numerically studied the effect of a low amplitude and periodic blowing and suction excitation through a narrow slot at the edge of the step on the mean recirculation length. Creusé and Mortazavi [7] numerically investigated the recirculation areas obtained by an inlet pulsed velocity in a laminar configuration. 
A closed-loop control by local forcing near the separation point was studied by Yi et al [30]: Using an adaptive sliding controller based on a proper orthogonal decomposition, a very significant reduction in the kinetic energy of the fluctuations was achieved. Two other very interesting references correspond to the turbulent channel flow control. Even if the configuration is no more the back-facing step, the flow control procedure can be used in a similar way to confined flow problems and to the separation control. The first of them is Hammond et al [13], which succeeded in attenuating near wall turbulence and reducing drag in wall bounded turbulent flows. It consists in blowing and suction at the wall, in the opposite direction to the wall normal fluid velocity. The direction is recorded respecting the flow unsteadiness at every time step in the vicinity of the boundary. The second is the one of Joshi et al [14], which developed feedback controllers based on linear theory that stabilize a two-dimensional plane Poiseuille flow to infinitesimal disturbances, using also a blowing/suction device to the wall in the normal direction for a laminar flow configuration.

The aim of the present work is to develop two different active control strategies to manipulate the flow characteristics inside a backward-facing step channel with a transitional flow regime: 1) using pulsed inlet velocities, with open and closed-loop frequency choices; 2) implementing two vertical jets to the vertical step wall with passive, open-loop or closed-loop action into the lower and upper levels of the step. This work follows the primary results obtained by the authors in a previous paper, devoted to the open-loop control of laminar flows in a similar geometry [7]. In this work, the impact of the control on several significant flow characteristics like vorticity, energy, fluctuations, velocity gradients etc. are carefully analysed.

The numerical simulations are performed using a Vortex-In-Cell method [5]. In VIC calculations, an Eulerian grid can be implemented in order to compute efficiently the velocity field on the Lagrangian particles. The goal is to obtain a fast computation of the particle velocities in regular bounded domains. VIC methods for viscous flows may be viewed as an appealing alternative to pure grid-free vortex methods in simple geometries. In this case, the use of fast Poisson solvers also enables fast velocity evaluations. Compared with pure finite-difference methods, VIC methods offer the advantage of a robust and accurate treatment of the convective part of equations with time steps not constrained by convective CFL conditions [6]. Moreover, the diffusion part of equations are solved by the Random Walk Algorithm [12] that is easy to implement with very small CPU time consuming and well adaptable to the Lagrangian particles transport [25], [22], [26]. 
In the following we describe the flow simulation method with some validation tests. This section covers the flow configuration, the governing equations, and the numerical code used for the simulation. The uncontrolled simulation is then validated, by comparing our results to some other numerical experiments available in the literature for two different Reynolds number ([26],[29]). The second part explains the control objectives in term of functionals definitions as well as different control strategies: open-loop control, closed-loop control with intrusive or non intrusive sensors. The next part is devoted to discussion on controlled numerical results related to the first approach (oscillating inlet velocities), to find which procedure is the most relevant in order to make the control efficient. Finally, in the last part the second control strategy (jets normal to the step wall) is numerically implemented and discussed in details. The results are then compared to each other.

\section{Flow simulation and validation}

\subsection{Flow configuration and governing equations}

The dynamics of a two-dimensional incompressible flow over a backward-facing step is governed by the conservation of mass and momentum, namely the twodimensional incompressible Navier-Stokes equations, given by :

$$
\begin{aligned}
\nabla \cdot \mathbf{u} & =0 \quad \text { in } \Omega \\
\frac{\partial \mathbf{u}}{\partial t}+\mathbf{u} \cdot \nabla \mathbf{u} & =-\nabla p+\frac{1}{R e} \Delta \mathbf{u} \quad \text { in } \Omega
\end{aligned}
$$

where $\Omega$ is the computational domain of Figure 1 with $L_{\text {down }}=10, H_{\text {step }}=0.5$

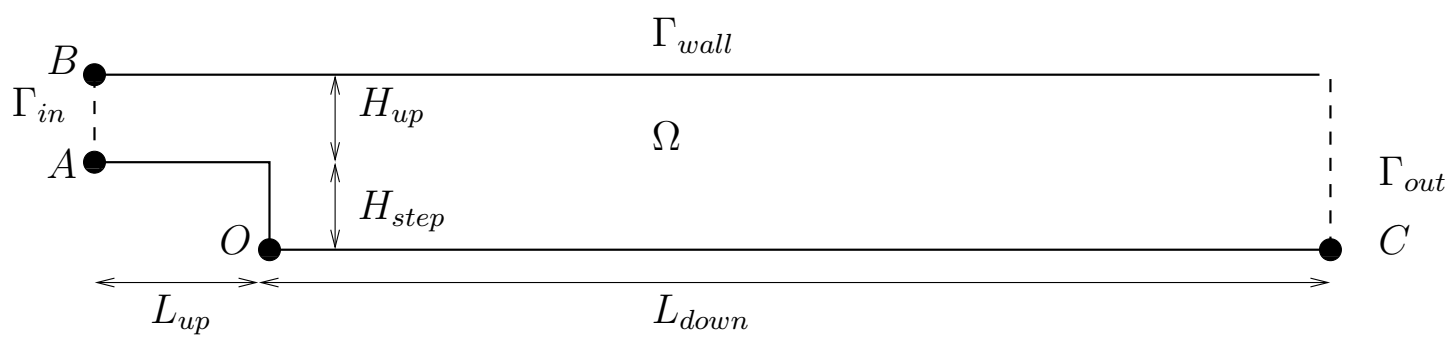

Fig. 1. The computational domain

and $H_{u p}=0.5$. According to the validation objective, we will set either $L_{u p}=1$ (similarly to [26]) or $L_{u p}=5$. The boundary conditions to impose on the wall $\Gamma_{\text {wall }}$ as well as on the inflow $\Gamma_{\text {in }}$ and on the outflow $\Gamma_{\text {out }}$ will be specified below. Here, $\mathbf{u}=(u, v)$ is the velocity normalized with respect to a given velocity $U, \mathbf{x}=(x, y)$ the two orthogonal space directions normalized with respect to $2 H$ where $H$ is the physical height of the step (that's why the computational 
height is $\left.H_{\text {step }}=0.5\right), t$ the time normalized with respect to $2 H / U$, and $p$ the pressure normalized with respect to $\rho U^{2}$ where $\rho$ is the density of the fluid. The numerical Reynolds number $R e$ is then defined by $R e=2 \rho U H / \mu$, with $\mu$ the dynamic viscosity of the fluid, which corresponds to a physical Reynolds number $R e_{\phi}=\rho U H / \mu=R e / 2$. The commonly used differential operators are given by:

$$
\nabla \cdot\left(f_{1}, f_{2}\right)^{T}=\frac{\partial f_{1}}{\partial x}+\frac{\partial f_{2}}{\partial y}, \nabla f=\left(\frac{\partial f}{\partial x}, \frac{\partial f}{\partial y}\right)^{T}, \Delta f=\frac{\partial^{2} f}{\partial x^{2}}+\frac{\partial^{2} f}{\partial y^{2}} .
$$

The vorticity $\omega$ is defined in two dimensions by the curl of the velocity vector :

$$
\omega=\nabla \times \mathbf{u}=\frac{\partial v}{\partial x}-\frac{\partial u}{\partial y} .
$$

Taking the curl of equation (2) and using equation (1), we get the Helmholtz or the Vorticity Transport Equation (VTE):

$$
\frac{\partial \omega}{\partial t}+\mathbf{u} \cdot \nabla \omega=\frac{1}{R e} \Delta \omega \text { in } \Omega
$$

expressing the transport of vorticity by convection and diffusion. Solving this equation permits us to convect and diffuse the vorticity field. Once the vorticity field known, integrating the equations (1) and (3) permits to describe the velocity field. The unsteady structure of these equations permits to find out the solution along the time. Finally, because the VTE doesn't contain directly the pressure term (taking the curl of Navier-Stokes equations this term is vanished), the pressure can be recovered from the velocity field by integrating the following Poisson equation:

$$
\Delta p=2\left(\frac{\partial u}{\partial x} \frac{\partial v}{\partial y}-\frac{\partial u}{\partial y} \frac{\partial v}{\partial x}\right) \quad \text { in } \Omega
$$

which is derived as the divergence of equation (2) also using the equation (1).

\subsection{Numerical method}

The previous equations are approximated using a Vortex method [3], [6]. These methods that are very robust and low-cost to simulate high Reynolds number recirculating flows (see for example [11], [26]), are more recently used to implement control techniques in fluid mechanics [24]. In this kind of approach, the VTE equation (4) is solved using a two-fractional step (or viscous splitting) method. It corresponds to approximate separately the diffusion and convection 
terms at each time step. Beale and Majda [2] prooved that the viscous splitting method converges towards the solution of the Navier-Stokes equations as the viscosity increases. The two fractional steps are:

$$
\begin{aligned}
\frac{\partial \omega}{\partial t}+\mathbf{u} \cdot \nabla \omega & =0, \\
\frac{\partial \omega}{\partial t} & =\frac{1}{R e} \Delta \omega .
\end{aligned}
$$

The convective part is solved using a "Vortex-In-Cell (VIC)" method (see e.g. [6]) with a semi-Lagrangian resolution. In this fractional step, the transport of vorticity due to convection is obtained from the solution of equation (6) in terms of the Lagrangian displacement of a set of finite vortex elements. If we define the stream function $\Psi$ by :

$$
u=\frac{\partial \psi}{\partial y} \text { and } v=-\frac{\partial \psi}{\partial x}
$$

and then substituting it in the equation (3), the following Poisson equation is achieved :

$$
-\Delta \psi=\omega
$$

Let assume that a number $n v^{n}$ of the finite vortex elements, located at $\mathbf{x}^{n}(i t)$ with a circulation (strength) $\gamma^{n}(i t)$, are known at time $t^{n}, 1 \leq i t \leq n v^{n}$. Let also assume that the vorticity field $\omega^{n}(i, j)$ at time $t^{n}$ is known on a cartesian mesh of the computational domain, $i$ and $j$ being respectively the horizontal and vertical indices of the mesh. The main target of the resolution method is to compute the same quantities at time $t^{n+1}=t^{n}+\delta t$ as $n v^{n+1}, \mathbf{x}^{n+1}(i t)$, $\gamma^{n+1}(i t)$ and $\omega^{n+1}(i, j)$.

In the first step of the computational procedure, the equation (9) with associated boundary conditions (see paragraph 2.3) is solved to recover the stream function field $\Psi^{n}(i, j)$. Then, solving the equation (8), the velocity $\mathbf{u}^{n}(i, j)$ is computed at each node of the mesh. Finally, using a linear interpolation procedure, a convective velocity $\mathbf{u}_{v}^{n}(i t)$ is associated to each finite vortex element $\left(1 \leq i t \leq n v^{n}\right)$, and so a convective displacement is given by $\mathbf{d l}_{\text {conv }}^{n}(i t)=\mathbf{u}_{v}^{n} \delta t$.

In the second fractional step, the solution of the equation (7) is simulated stochastically with the displacement of the vortex elements by the random walk method. This method is based on the theorem expressing that the brownian motion of an infinite number of particles converges towards the solution of the heat equation ([3], [12]). The Green function of the two-dimensional 
diffusion equation (7) is given by:

$$
G r(x, y, t)=\frac{R e}{4 \pi t} \exp \left(-\frac{R e\left(x^{2}+y^{2}\right)}{4 t}\right)
$$

which is identical to the probability density fonction of a Gaussian random variable $\eta=\left(\eta_{x}, \eta_{y}\right)$ with a zero mean and a standard deviation $\sigma(t)=$ $\sqrt{2 t / R e}$ :

$$
G\left(\eta_{x}, \eta_{y}, t\right)=\frac{1}{2 \pi \sigma^{2}(t)} \exp \left[-\frac{1}{2 \sigma^{2}(t)}\left(\eta_{x}^{2}+\eta_{y}^{2}\right)\right]
$$

where $\eta_{x}$ and $\eta_{y}$ are two independant random variables related to directions $x$ and $y$. Based on the linearity of equation (7), the solution of (7) is simulated stochastically by 2-dimensional displacement of the vortex elements in perpendicular directions $x$ and $y$ using two sets of independant Gaussian random numbers with a zero mean and $\sigma=\sqrt{2 \delta t / R e}$ standard deviation . This leads to a random and markovian Lagrangian displacement of $\mathbf{d l}_{\text {diff }}^{n}($ it $)$ from time $t^{n}$ to time $t^{n+1}$.

Then, the final location of each finite vortex element $\mathbf{x}_{*}^{n+1}(i t)$ at time $t^{n+1}$ is obtained as the sum of the convective and diffusive movements during the last time step:

$$
\mathbf{x}_{*}^{n+1}(i t)=\mathbf{x}^{n}(i t)+\mathbf{d l}_{\text {conv }}^{n}(i t)+\mathbf{d l}_{\text {diff }}^{n}(i t) .
$$

If the vortex element leaves the computational domain through the inlet or outlet boundary, then the vortex is destroyed and does not exist any more in the domain. However, because the lagrangian property of vortex methods there is a very small probability that a computational particle leaves the domain by a solid wall. Of course, such an event is not natural and is related to the computational errors in the fluid trajectory especially because the random walk method ([26], [12]). In this case, the vortex element has to be replaced in the computational domain. To achieve this aim, the exited particle is reflected to the internal domain through the solid wall. This reflection procedure is symmetrical respecting to the wall position. It should be outlined that this element "recapturing" approach is only valid for a very small number of exhausted elements and the computation is interrupted if the number of exited vortices via solid walls becomes higher than an accuracy limit ([3], [6]).

In incompressible flows the unique source of generation of the vorticity is the no-slip boundary condition. Vortex methods mimic this physical phenomenon. So, the slip velocity on the wall is nullified by the generation of new vortex 
elements on the boundary [4]; it also compensates the vorticity leaving the domain from the exit boundary. Therefore, the last step of the algorithm is devoted to the vortex generation on the solid boundary. The task is performed using an induced circulation cancelling of the slip velocity $\mathbf{u}^{n}(i, j) . \tau$ computed in the first step on the nodes of the wall boundary, where $\tau$ is the tangential vector to the wall. New vortices are then injected in the domain by the normal diffusion. The number of newly created vortices is determined by the value of the slip velocity in order to achieve a zero velocity at each boundary point. The new number of vortices $n v^{n+1}$ as well as their location $\mathbf{x}^{n+1}(i t)$ and circulation $\gamma^{n+1}(i t)$ is then known $\left(1 \leq i t \leq n v^{n+1}\right)$.

The circulation of vortices is finally distributed on the grid nodes, allowing to recover the vorticity field $\omega^{n+1}(i, j)$ for the post-processing needs, and to repeat the whole procedure to get the variables at the following time steps.

\subsection{Boundary conditions}

To close the problem, boundary conditions on $\mathbf{u}$ have to be added to the set of equations (1)-(2), which are :

$$
\begin{aligned}
\mathbf{u} & =(0,0)^{T} & & \text { on } \quad \Gamma_{\text {wall }}, \\
\mathbf{u} & =(1,0)^{T} & & \text { on } \quad \Gamma_{\text {in }}, \\
\frac{\partial v}{\partial x} & =0 \quad \text { on } & & \Gamma_{\text {out }} .
\end{aligned}
$$

Boundary conditions (13) and (14) are very common. The choice of $u=1$ for the boundary $\Gamma_{i n}$, means that the normalizing velocity $U$ introduced in the section 2.1 corresponds to the physical inlet velocity. The boundary condition (15) is chosen for its accuracy and simplicity to implement in vortex techniques [20]. In vortex methods, boundary conditions are only used to solve the Poisson equation (9), and have to be expressed in terms of the non-primitive variable $\Psi$. Since $\Psi$ has a constant variation from the lower to the upper wall, we set $\Psi(O)=0$, with $O$ as the origin of the axis (see Figure 1). Therefore, on the bottom part of $\Gamma_{\text {wall }}$, the boundary condition (13) leads to $\Psi=0$. On $\Gamma_{i n}$, the boundary condition (14) leads to $\partial \Psi / \partial y=1$, that also means $\Psi(-1, y)=y-H_{\text {step }}$ since $\Psi(A)=0$. On the upper part of $\Gamma_{\text {wall }}$, the boundary condition (13) reads $\Psi=\Psi(B)=H_{u p}-H_{\text {step }}$. Finally, on $\Gamma_{\text {out }}$, the boundary condition (15) is resumed as $\partial^{2} \Psi / \partial x^{2}=0$. 


\subsection{Validation of the simulation}

The validation of the simulation is performed for two Reynolds numbers : $R e=500\left(R e_{\Phi}=250\right)$, and $R e=2000\left(R e_{\Phi}=1000\right)$. The first flow corresponds to a low transitional regime. It is motivated by the fact that our results can be compared to existing literature [26] on the same geometry $\left(L_{u p}=1\right)$ for several characteristic quantities : The length of the averaged recirculation area, the streamwise velocity profiles, the time trace of of the streamwise velocity at several monitoring points in the flow. Some computations are also performed on a geometry with $\left(L_{u p}=5\right)$, to make the comparisons possible with other bibliography references concerning the length of the averaged recirculation area $([1,15,19,9,23])$. We should also outline that for the first Reynolds number, several experiments to validate the vortex computations are available.

The second one corresponds to a higher transitional regime, and constitutes the reference flow to control in the following of the paper. For this case, the validation is ensured by the verification of the grid convergence property on the length of the averaged recirculation area as well as the comparisons with the literature [29] concerning the frequency behavior related to the control strategy. Here, even if the flow is transitional with the onset of instabilities, our validations respect to other studies, permit to build a coherent control benchmark that can be used in a generic way. The main target is to perform a large number of control simulations in order to develop a useful data base on the backward-facing step active control. Such a source can be obtained by the two-dimensional case studies.

\subsubsection{Validation at $R e=500$}

Three sets of discretization parameters are used in vortex methods. These parameters are summarized in Table 1 . The parameters $n x$ and $n y$ are respectively, the number of mesh nodes in the horizontal and vertical directions. $C_{\text {max }}$ is the elementary circulation value of a vortex element, and $\delta t$ the time step. Previous studies have shown that an appropriate choice of these parameters considering their mutual influence on each other is necessary in order to achieve accurate and low-cost computations ([25], [22], [21]).

Taking $h=\left(L_{u p}+L_{\text {down }}\right) / n x=\left(H_{\text {step }}+H_{u p}\right) / n y$, the values of parameters $C_{\max }$ and $\delta t$ with respect to $h$ from coarse (level 1) to fine grids (level 3) are chosen with a linear evolution. This property was inspired from the work of Pellerin [25] for a similar vortex method, and also from studies of Mortazavi et al. [22], [21], who studied the dependence between these three discretization parameters for a purely lagrangian vortex approach. 
Table 1

\begin{tabular}{|l||c|c|c|}
\hline & Level 1 & Level 2 & Level 3 \\
\hline \hline$n x$ & $150+15 L_{u p}$ & $300+30 L_{u p}$ & $600+60 L_{u p}$ \\
\hline$n y$ & 15 & 30 & 60 \\
\hline$C_{\text {max }}$ & $2 \mathrm{E}-04$ & $1 \mathrm{E}-04$ & $5 \mathrm{E}-05$ \\
\hline$\delta t$ & $2 \mathrm{E}-01$ & $1 \mathrm{E}-01$ & $5 \mathrm{E}-02$ \\
\hline
\end{tabular}

The three sets of parameters.

Table 2

\begin{tabular}{|c|c|c|c|}
\hline $\begin{array}{c}\text { Min. / Max. values of } \overline{L_{r}} \\
\text { in }[1,15,19,9,23]\end{array}$ & Level 1 & Level 2 & Level 3 \\
\hline $4.16 / 5.97$ & 3.63 & 4.71 & 4.62 \\
\hline
\end{tabular}

The averaged recirculation length $\overline{L_{r}}$ values for $R e=500\left(R e_{\phi}=250\right), L_{u p}=5$.

At $t=0$, there is no vortices in the domain and the velocity is equal to zero excepted at the inlet (condition (14)). The calculation is performed from $t=0$ to $t=600$, and to obtain a well established flow the time-averaged quantities are processed from $t=300$ to $t=600$.

A first set of tests is performed using $L_{u p}=5$. Here, the inlet section is far enough from the step to provide a fully developped parabolic velocity profile just in front of the step. Results are compared to the ones obtained by many other papers $([1,15,19,9,23])$ on the averaged recirculation length $\overline{L_{r}}$. Table 2 gives the value of $\overline{L_{r}}$ obtained by the computations in this work, as well as its lowest and highest values obtained in previously mentionned works for the same Reynolds number. We can see that using level 1 computations the value of $\overline{L_{r}}$ is very different from the litterature, but the results are in good agreement with the available literature for levels 2 and 3 .

A second set of tests is then performed using $L_{u p}=1$ to be compared to Sethian and Ghoniem ([26]). In this case, the parabolic velocity is not entirely developped at the step position. Figure 2 shows the averaged recirculation area for each of the three sets of discretization parameters, and Table 3 gives the corresponding value of $\overline{L_{r}}$, comparing it to the value obtained in [26]. Then, Figure 3 shows the averaged velocity profiles at $x=1, x=2, x=3, x=4$, $x=5$ and $x=6$, again compared to Sethian and Ghoniem.

Results obtained in Table 3 with levels 2 and 3 are in very good agreement with the corresponding results in [26] (less than $0.8 \%$ of relative error). The grid convergence is achieved from level 2, since the results obtained with Level 2 and Level 3 on Figures 2 and 3 are very close to each other (on Figure 3, the dotted line (level 2) can not been distinguished from the plain one (level 3). 
Table 3

\begin{tabular}{|c|c|c|c|c|}
\hline & Ref. [26] & Level 1 & Level 2 & Level 3 \\
\hline$\overline{L_{r}}$ & 3.93 & 3.50 & 3.95 & 3.96 \\
\hline
\end{tabular}

The averaged recirculation length $\overline{L_{r}}$ values for $R e=500, L_{u p}=1$.

Figure 4 shows the time history of the horizontal component of the velocity $u$ from $t=40$ to $t=90$ at three different monitoring points $M_{1}=(8.0,0.2)$, $M_{2}=(8.0,0.5)$ and $M_{3}=(8.0,0.8)$, for every discretization parameter set. For all of the three different parameter sets, the averaged value of $u$ is larger in $M_{2}$ than in $M_{1}$ and $M_{3}$. It corresponds to the fact that the motion of some eddies are more sharper near the top and bottom walls than in the middle of the section. The same behavior was underlined in [26].
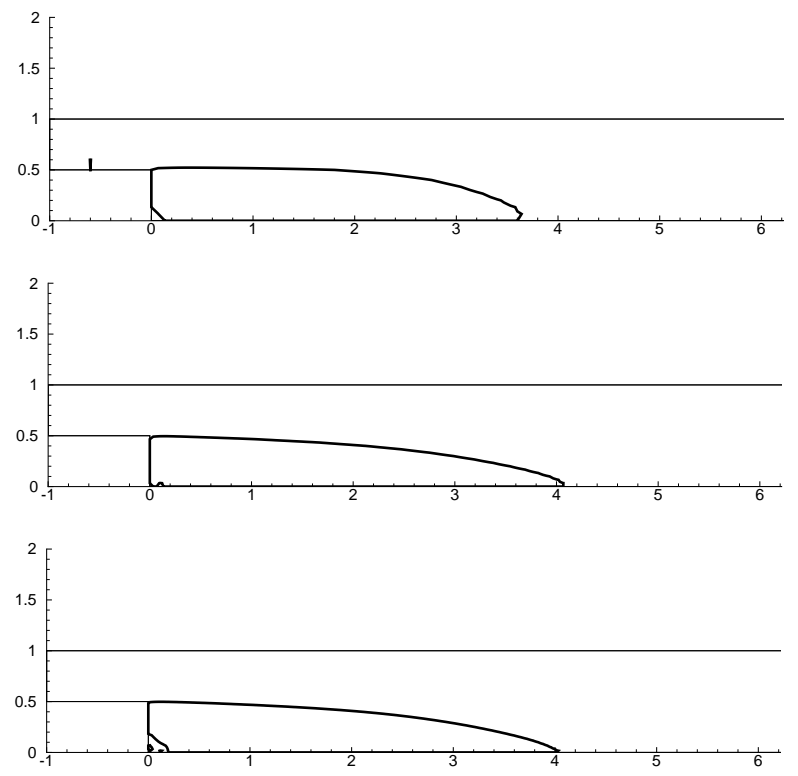

Fig. 2. Averaged recirculation areas for $R e=500$ : Isovalue $\Psi=0$. Top : Level 1, Middle : Level 2, Bottom : Level 3.

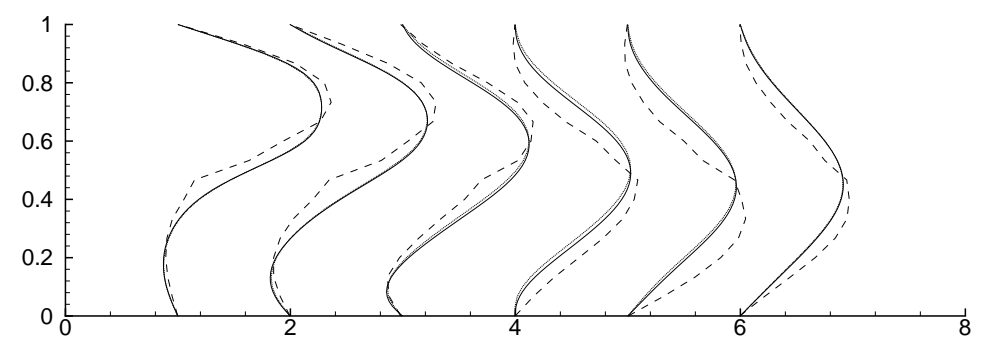

Fig. 3. Averaged streamwise velocity profiles for $R e=500$. Dashed : Level 1, Dotted : Level 2, Plain : Level 3. 

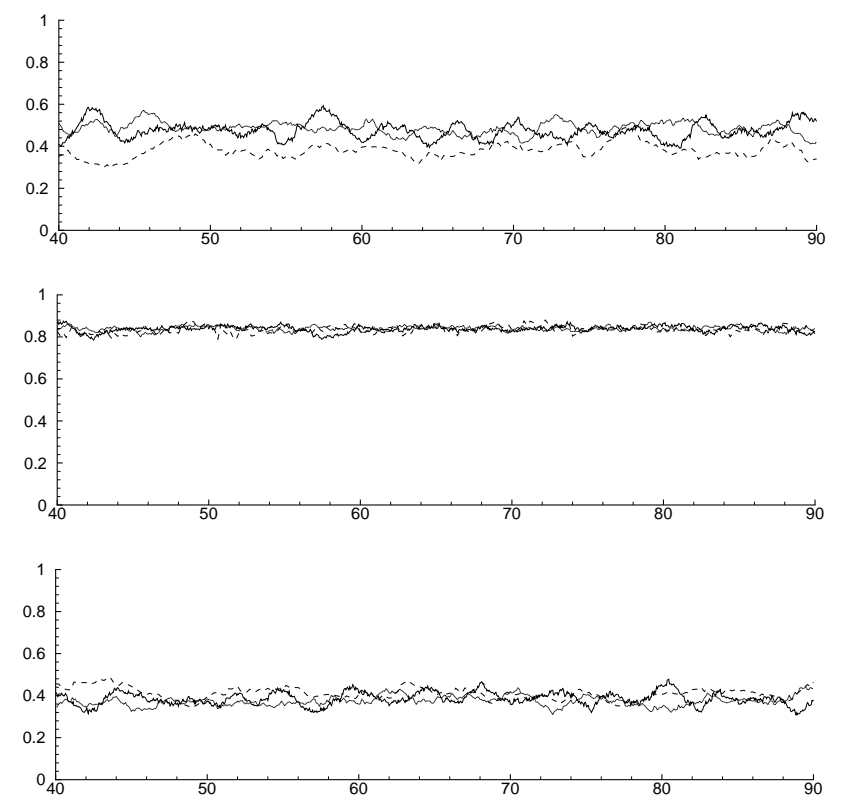

Fig. 4. $u$ for $R e=500$ from $t=40$ to $t=90$ at points $M_{1}$ (top), $M_{2}$ (middle) and $M_{3}$ (bottom). Dashed : Level 1, Dotted : Level 2, Plain : Level 3.

Table 4

\begin{tabular}{|c|c|c|}
\hline & Level 2 & Level 3 \\
\hline$\overline{L_{r}}$ & 4.58 & 4.39 \\
\hline
\end{tabular}

The averaged recirculation length $\overline{L_{r}}$ values for $R e_{\phi}=1000, L_{u p}=1$.

This first validation leads to the conclusion that for $R e=500$, level 1 fails to give a sufficiently accurate solution whereas level 2 and level 3 ensure very good results compared to the litterature references. Consequently, the validation at $R e=2000$ will be performed using only level 2 and level 3 .

\subsubsection{Validation at $R e=2000$}

A second validation is now performed at $R e=2000$ in the high transitional regime, in order to obtain a more unsteady flow, which will be the reference flow to control. In order to preserve -at least partially- the initial aspect of the inlet flow, we set $L_{u p}=1$. In fact, because the physical target of this paper is to implement control tools into the inlet flow (see next section), the upstream channel is chosen as short as possible to avoid the dissipation of these effects before the step. The table 4 gives the corresponding values of $\overline{L_{r}}$ for the two parameter sets. The obtained results are very close to each other for both discretizations levels (less than $4 \%$ of relative error).

When the regime is established, a spectral analysis of $\frac{\partial^{2} u}{\partial y^{2}}$ as a function of 
Table 5

\begin{tabular}{|l||c|c|}
\hline Dominant frequency & Level 2 & Level 3 \\
\hline \hline Sensor $x=6$ & 0.1367 & 0.16 \\
\hline Sensor $x=7$ & 0.1367 & 0.1267 \\
\hline Sensor $x=8$ & 0.1367 & 0.1267 \\
\hline
\end{tabular}

Dominant frequency at $R e=2000$ for sensors located at $x=6, x=7$ and $x=8$.

the time is performed for Level 2 , at 3 sensors on the bottom wall $(y=0)$ respectively located at $x=6, x=7$ and $x=8$ (see Figure 5 ). The choice of this particular physical quantity to record by the sensors is justified below. For each of these sensors, the dominant frequency of the signal corresponds to a Strouhal number close to 0.067 (this frequency is equal to $2 \mathrm{St}$ since $\left.H_{\text {step }}=0.5\right)$. This result is the same as the one obtained in Wee et al $([29])$ : The absolute mode frequencies corresponding to the downstream region are all in the order of $S t \sim 0.07$ that is the standard Strouhal number for this range of Reynolds numbers in channel flows. The slow variation of the absolute mode frequency is directly due to the slow change of the shear layer thickness in the region close to the end of the recirculation zone. As the table 5 indicates, this dominant frequency is captured by every sensor (especially for the two sensors located at the highest $x$-values $(x=7$ and $x=8)$, whatever the used discretization grid level. So, the grid convergence is achieved for $R e=2000$.
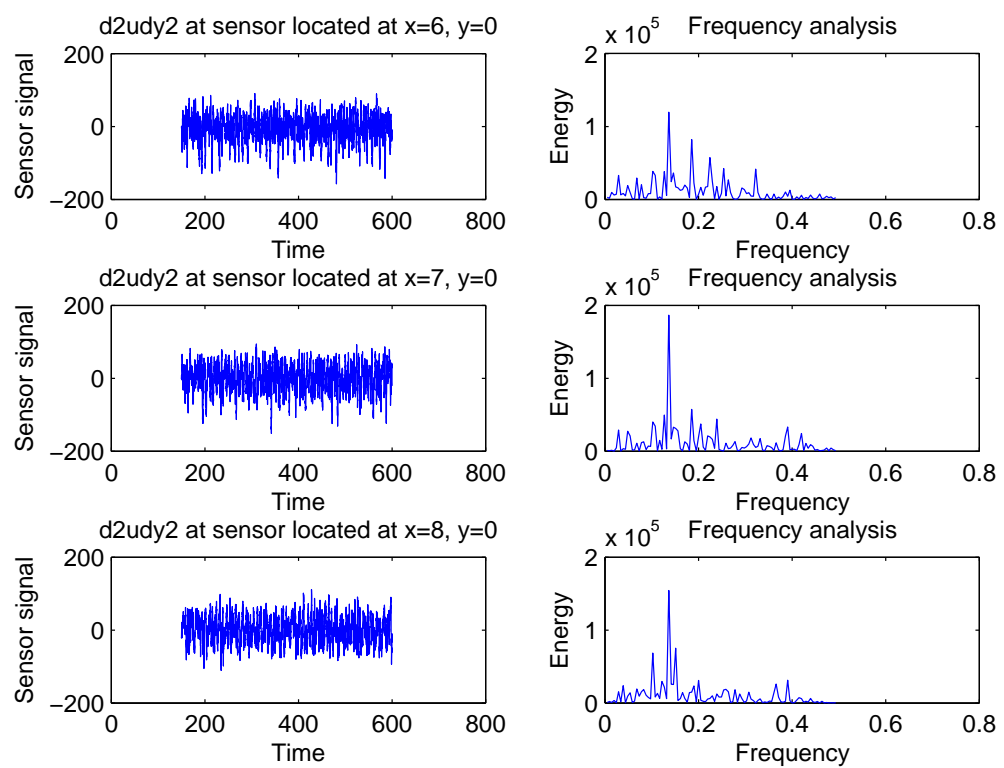

Fig. 5. Signal of $\frac{\partial^{2} u}{\partial y^{2}}(t)$ for $R e=2000$ at intrusive sensors on the bottom wall at $x=6, x=7$ and $x=8$ for level 2 and spectral analysis.

Based on the above validations and the numerical convergence results, all the numerical tests in forthcoming sections are performed on the level 2 discretiza- 
tion parameter zone. This level is not only fine enough to get accurate results, but also needs a reasonable computational cost in order to explore efficient control strategies with a large number of numerical experiments. A simulation on the level 2 grid at $R e=2000$ generates about $n v=2.5 .10^{6}$ vortices with $\delta t=0.1$ and a quite fast establishment of the number of discrete particles towards a plateau confirming the convergence of the calculations [22]. Nevertheless, the same simulation on a level 3 needs more than $6.5 .10^{6}$ particles with a twice smaller time step, therefore a slower initial establishment and a CPU time six times larger than for level 2. These observations confirm our

choice to use the level 2, as a good compromise between the accuracy and a low computational cost.

\section{Control}

In this section different active control tools are designed and studied in order to manipulate the main recirculation zone developped behind the step wall. Among the control targets we can mention the reduction of the recirculation area or to shorten the reattachment length. This procedure is directly related in modifying the vortex shedding on the step and then altering the vortex dynamics inside the channel. This control can also permit to reduce or increase the residence time of trapped particles inside the recirculation zone. It should be outlined that we focus in this work on the control of the vortex shedding and the vorticity field mainly generated by the step effect and its recirculation zone. We don't include the secondary vortices which are generated close to the upper wall without any important effect on the shedding dynamics.

The control is performed either by taking an oscillating inlet velocity $\mathbf{u}(t)=$ $\left(u_{i n}(t), 0\right)^{T}$ on $\Gamma_{i n}$, instead of the uniform velocity boundary condition (14) associated to the non-controlled simulation (Figure 6, Control 1 ), or by two small jets implemented on the upper and lower parts of the step (Figure 6, Control $_{2}$ ).

Both, open loop and closed-loop controls are used and compared to each other. The open loop control studies are performed in both cases for a wide range of parametric values to get the most efficient control configurations. These values are then compared to the closed-loop control simulations to verify its efficiency for every control problem. Since the value of the inflow velocity is no more constant in time, boundary condition on $\Psi$ on the inflow boundary as well as on the top boundary, needed for the resolution of (9), is updated at each computational time step. 


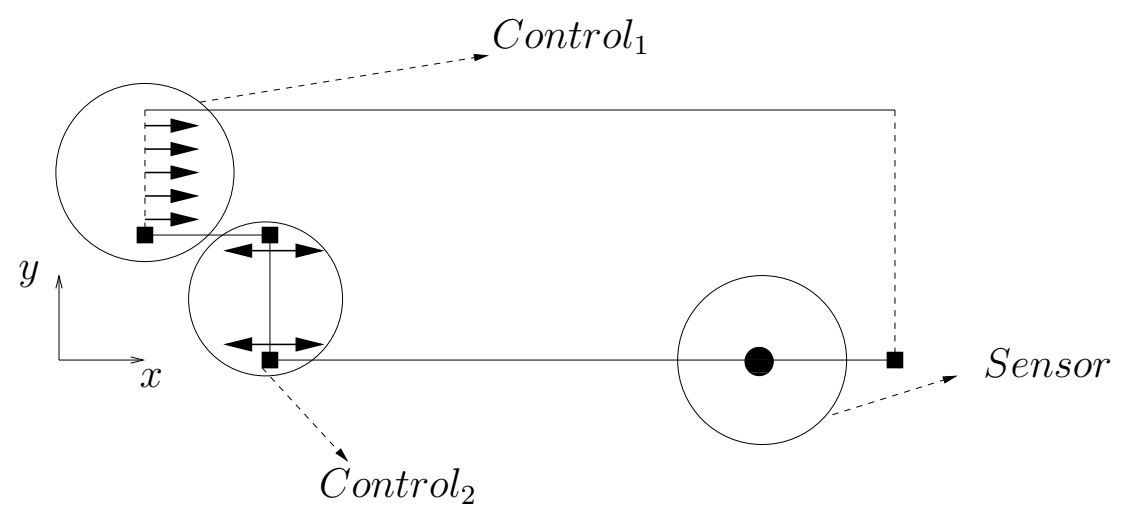

Fig. 6. Control devices on the step geometry.

\subsection{Control functionals and devices}

As mentioned before, the control effect is focused on the vortex shedding, transport phenomena and the step recirculation zone behavior. In order to quantify this control, the following time dependent fonctionnals are introduced :

(1) The recirculation area length $L_{r}(t)$. This functional is the most common physical quantity to characterize the backward-facing step flow. It is defined as the distance between the point $O$ (Figure 1) and the point located on the bottom wall for which the normal gradient of the horizontal velocity component is equal to zero and after reachs a positive value;

(2) The total enstrophy in the domain that measures the vorticity of the flowfield, defined as:

$$
J_{\omega}(\mathbf{u}(t))=\frac{1}{2} \int_{\Omega} \omega^{2}(t) d t
$$

where $\omega$ is defined by (3).

(3) The total kinetic energy in the domain. One of the control tasks is to tune the energy of vortical structures. It is defined as:

$$
J_{E}(\mathbf{u}(t))=\frac{1}{2} \int_{\Omega} u^{2}(t)+v^{2}(t) d t .
$$

The control has to reduce the entrophy and energy of the flow.

(4) The near wall recirculation area intensity that corresponds to the level of shear forces defined by:

$$
J_{W}(\mathbf{u}(t))=\int_{0}^{C} \frac{\partial u}{\partial y}(t)\left(\frac{\partial u}{\partial y}(t)-\left|\frac{\partial u}{\partial y}(t)\right|\right) d x
$$


Breaking the step-side large recirculation zone this functional may increase because the creation of smaller structures with high velocity gradient close to the wall.

(5) The fluctuation coefficient. This coefficient corresponds to the flow instabilities in transition towards turbulence deviating the flow from the mean values:

$$
J_{F}(\mathbf{u}(t))=\iint_{B} \frac{d x d y}{1+\frac{\left(u^{\prime}(t)\right)^{2}+\left(v^{\prime}(t)\right)^{2}}{2}},
$$

where $B$ is the box $[0 ; 6] \times[0 ; 0.5]$, and $u^{\prime}(t)\left(\right.$ resp. $\left.v^{\prime}(t)\right)$ is the variation of $u(t)$ (resp. $v(t)$ ) around its mean value. This functional is quite sensible to flow oscillations induced by the pulsing control strategy.

The above functionals will be used not only in their unsteady time evolution, but also for their following time-averaged values achieved between $t=T_{1}$ and $t=T_{2}$ who write as:

$$
\begin{array}{ll}
\overline{L_{r}}=L_{r}(\overline{\mathbf{u}}) & \overline{\overline{J_{F}}}=\frac{1}{T_{2}-T_{1}} \int_{T_{1}}^{T_{2}} J_{F}(\mathbf{u}(t)) d t \\
\overline{J_{\omega}}=J_{\omega}(\overline{\mathbf{u}}) & \overline{\overline{J_{\omega}}}=\frac{1}{T_{2}-T_{1}} \int_{T_{1}}^{T_{2}} J_{\omega}(\mathbf{u}(t)) d t \\
\overline{J_{E}}=J_{E}(\overline{\mathbf{u}}) & \overline{\overline{J_{E}}}=\frac{1}{T_{2}-T_{1}} \int_{T_{1}}^{T_{2}} J_{E}(\mathbf{u}(t)) d t \\
\overline{J_{W}}=J_{W}(\overline{\mathbf{u}}) & \overline{\overline{J_{W}}}=\frac{1}{T_{2}-T_{1}} \int_{T_{1}}^{T_{2}} J_{W}(\mathbf{u}(t)) d t
\end{array}
$$

with :

$$
\overline{\mathbf{u}}=\frac{1}{T_{2}-T_{1}} \int_{T_{1}}^{T_{2}} \mathbf{u}(t) d t .
$$

Our objective is to control these instantaneous and time-averaged functionals. In the following, the simulations are performed between $t=0$ and $t=600$. The control starts at $t=300$, and the time-averaged functionals are evaluated from $T_{1}=320$ and $T_{2}=600$.

\subsection{Control with pulsing inlet velocities}

In this part the control is achieved oscillating the inlet velocity profile: 


$$
u_{\text {in }}(t)=1.0+A \cos (2 \pi f t)
$$

The frequency $f$ and the amplitude $A$ of this oscillating flow can be obtained either by an extensive parametric study (open-loop control) or implementing the dominant frequencies of the vorticity formation and transport in the pulsing flow as the reference value (closed-loop control).

\subsubsection{Open-loop Control: Influence of the frequency $f$}

In this section the influence of the frequency $f$ on the flow behavior for a constant value of $A$ will be studied.

The value of amplitude is taken $A=0.2$, in the same order as the average fluctuations absolute value. For 50 low frequencies uniformly distributed in the range $[0.0 ; 1.0]$, the averaged functional values defined in part 3.1 are ploted as a function of the frequency $f$ (see Figure 7).
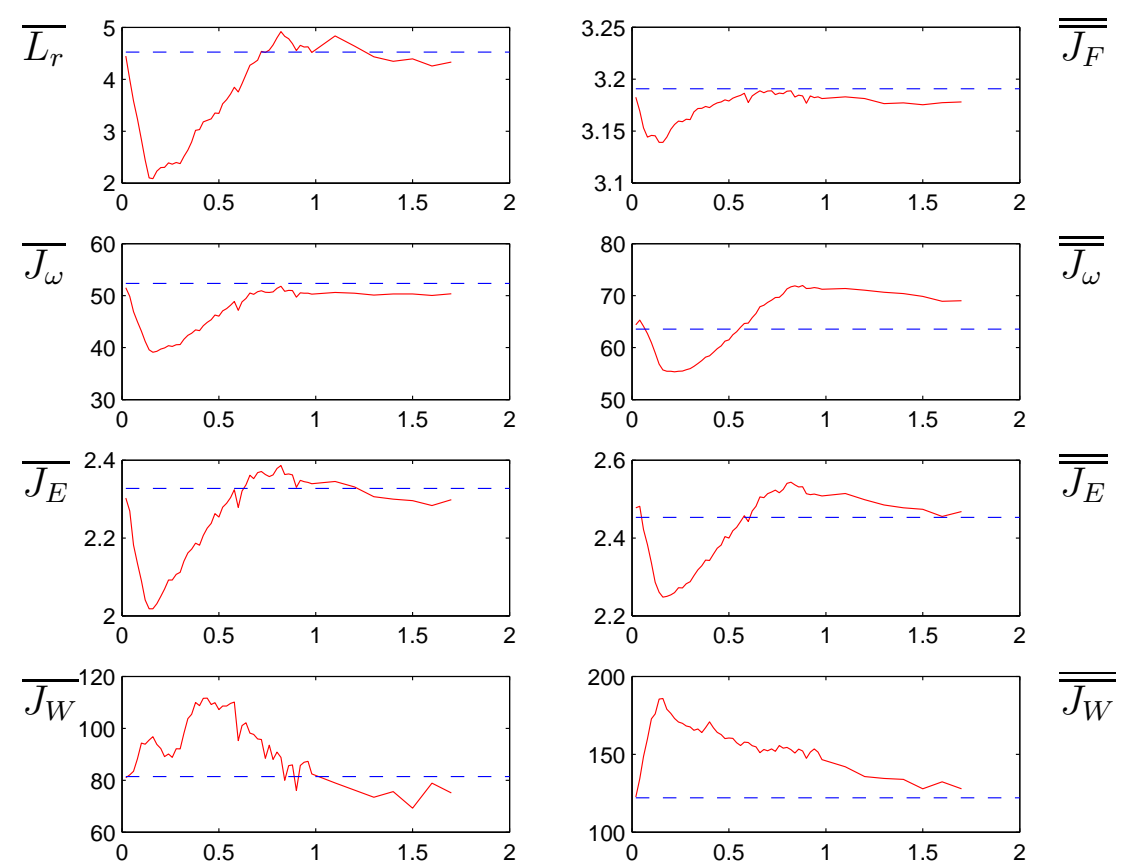

Fig. 7. Open-loop control: Functionals versus frequency. Dashed line: uncontrolled simulation

As the figure shows, an extremum value for each functional for a frequency nearly equal to the basic fundamental frequency of the flow, namely $f \approx 0.14$ 
is achieved. $\overline{L_{r}}$ as well as $\overline{J_{\omega}}$ (and $\overline{\overline{J_{\omega}}}$ ), $\overline{J_{E}}$ (and $\overline{\overline{J_{E}}}$ ) and $\overline{\overline{J_{F}}}$ are significatively diminished, and $\overline{\overline{J_{W}}}$ is consequently increased. It corresponds to the fact that the recirculation area is broken by this control strategy, and then the total enstrophy and energy of the flow descreases and is focused in the near wall small coherent structures. Nevertheless, the increase of $\overline{J_{W}}$ for the mean flow does'nt represent the control efficiency, because $J_{W}$ is more related to the instantaneous near-wall shear effects of vortical structures. That's why in the following we will only consider $\overline{\overline{J_{W}}}$ which is a more relevant quantity. For the larger values of the frequency, the efficiency of the control is reduced, but it becomes stable with a permanent effect on the flow behavior.

\subsubsection{Open-loop Control: Influence of the amplitude $A$}

The value of the frequency is taken $f=0.14$ (the basic fundamental frequency of the flow). For 10 amplitude values distributed in the range $[0.0 ; 1.0]$, we plot the averaged functional values defined in part 3.1 as a function of the frequency $A$ (see Figure 8).

This figure shows that the efficiency of the control almost linearly increases with the increasing values of the amplitude until $A=0.2$, attending then a "plateau". For larger values of amplitude the control effect doesn't change anymore. These results confirm the existence of an optimal control amplitude $A=0.2$ and there is no need to take higher $A$ values.

\subsubsection{Closed-loop control}

The closed-loop control consists in using the fundamental frequencies of flow shedding or transport in order to fit the oscillation frequency. The frequency measuring sensors may be implemented on the wall (non-intrusive sensors) or inside the flow field (intrusive sensors). The main advantage of the closed-loop control is that the control frequency is taken directly from the principal flow frequencies. Therefore, with a good choice of sensors, it is not only very simple to implement but also it avoids the heuristic trial and error type approaches necessary in open-loop methods.

\subsubsection{Non-intrusive sensors}

This first section is devoted to closed-loop control using non-intrusive sensors. Because the non-intrusive sensors are implemented in the channel walls and deal with the measure of the wall tangential pressure gradient, they are much 

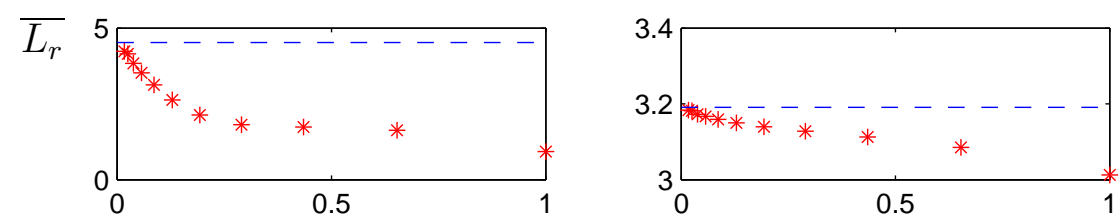

$\overline{\overline{J_{F}}}$
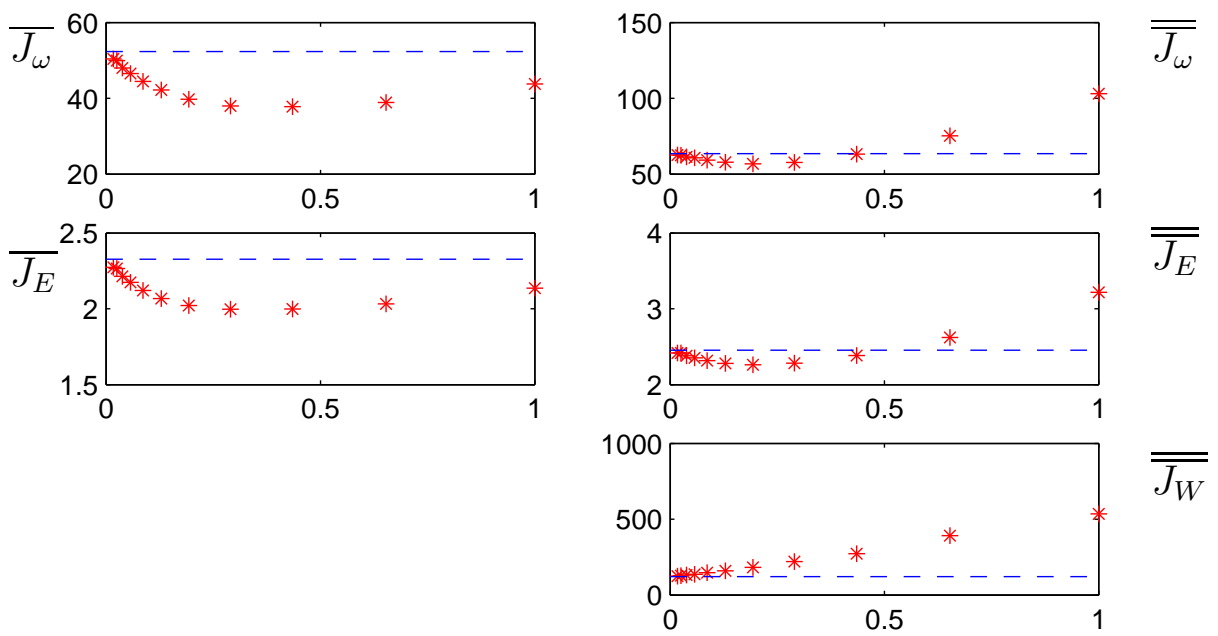

Fig. 8. Open-loop control: Functionals versus amplitude. Dashed line: uncontrolled simulation

more easier to be used in experimental and industrial applications. However, their near wall location makes them vulnerable to the noisy near-wall high gradient flow motion. From numerical point of view, because vortex methods approximate non-primitive variables, the pressure field is not directly obtained from computations. Therefore, the boundary layer models are used to connect the quantity $\partial^{2} u / \partial y^{2}$, to the pressure gradient. Indeed, in the wall since $\mathbf{u} \equiv 0$, the equation (2) is reduced to:

$$
\frac{\partial p}{\partial x}=\frac{1}{R e} \frac{\partial^{2} u}{\partial y^{2}}
$$

The right member of the equation (16) represents the Lighthill's conceptual model of vorticity generation at the wall [17], vorticity acquired by the fluid elements near the wall. As a consequence, on the wall, the signal $\partial^{2} u / \partial y^{2}(t)$ is equivalent to the signal $\partial p / \partial x(t)$.

Closed-loop control consists in taking:

$$
u_{i n}(t)=1.0+M\left(\partial^{2} u / \partial y^{2}(t)\right)_{S_{n}},
$$

where $M=2 \cdot 5 \cdot 10^{-3}$ is chosen to make vary $u_{\text {in }}(t)$ in the range of $[0.8 ; 1.2]$ as in section 3.2.2, and $\left(\partial^{2} u / \partial y^{2}(t)\right)_{S_{n}}$ is the value of $\partial^{2} u / \partial y^{2}(t)$ quantity related to a non intrusive sensor $S_{n}$. Five simulations are performed, using for each 
of them a different sensor locations $\left(x_{S_{n}}=3+n, y_{S_{n}}=0,1 \leq n \leq 5\right)$. These locations permit to get on the wall different dominant frequency values from the recirculation area until the downstream channel region. We plot the functional values as a function of $x_{S_{n}}$ on Figure 9. For each graph, we also plot the value of the functional values for the uncontrolled simulation (cf. part 2.4.2) as well as the best value obtained by the open-loop control (the optimal values observed for these functionals).
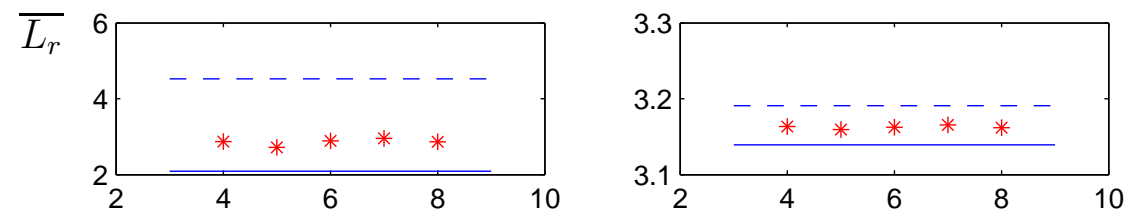

$$
\overline{\overline{J_{F}}}
$$
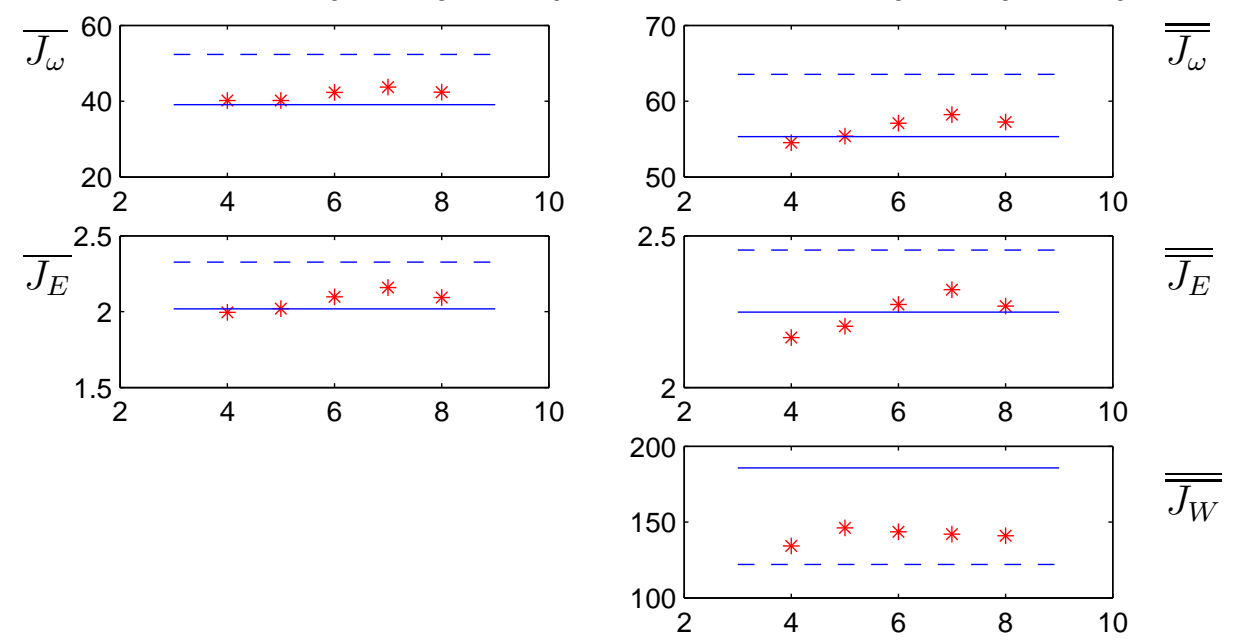

Fig. 9. Closed-loop control: Functionals versus $n$. Dashed line : uncontrolled simulation, solid line: best value obtained by open-loop control.

As Figure 9 shows, closed-loop control is efficient since each obtained functional value is close to the best result achieved by the open-loop control, whatever the sensor used. The important advantage of the closed-loop control is that automatically without a huge computational cost the backward-facing step flow is controlled. Especially, the sensor values recorded on the edge of the reattachment point are very interesting. As the figure shows, these points give an excellent recirculation length, fluctuation, energy and vorticity reduction. The wall stress forces for the mean flow also are reduced even if the average value of the shear forces is still larger than non-controlled flow. As mentioned before, this last behavior is because the control is achieved by the flow oscillation.

To understand the dynamics of the flow, instantaneous streamlines are ploted on figures 10 and 11 from time $t=592.5$ to $t=600$. It coresponds to a simulation time of 7.5 equivalent to fundamental period at basic control fre- 
quency $f=0.1367$. Figure 10 corresponds to the uncontrolled flow, and figure 11 to the controlled flow with the sensor located at $x_{S}=8$. Similarly, the instantaneous recirculation areas are shown on Figure 12 (without control) and figure 13 (with control). As the figures show the recirculation zone area is considerably reduced in the controlled case and replaced by two small counterrotating vortex structures. The shedding mechanism il also changed: the upper clockwise structure breaks the lower counter-clockwise one and creates a shorter shedding process, and generates small faster traveling vortices. Finally, the efficiency of this closed-loop control is verified plotting the mean streamlines and the mean recirculation areas, and comparing them to mean flows in uncontrolled cases (Figures 14 and 15). As the figures show the averaged recirculation zones are remarquably reduced and concentrated in a small area behind the step using the closed-loop control. That means that with control less particles will be trapped in the main recirculation region. 

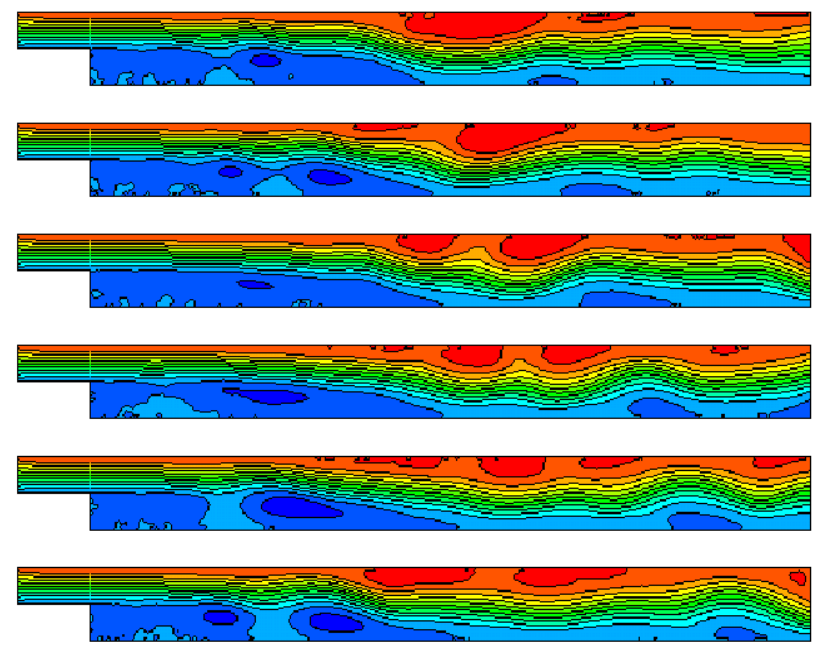

Fig. 10. Streamlines from $t=592.5$ to $t=600.0$, with $\Delta t=1.5$, uncontrolled regime.

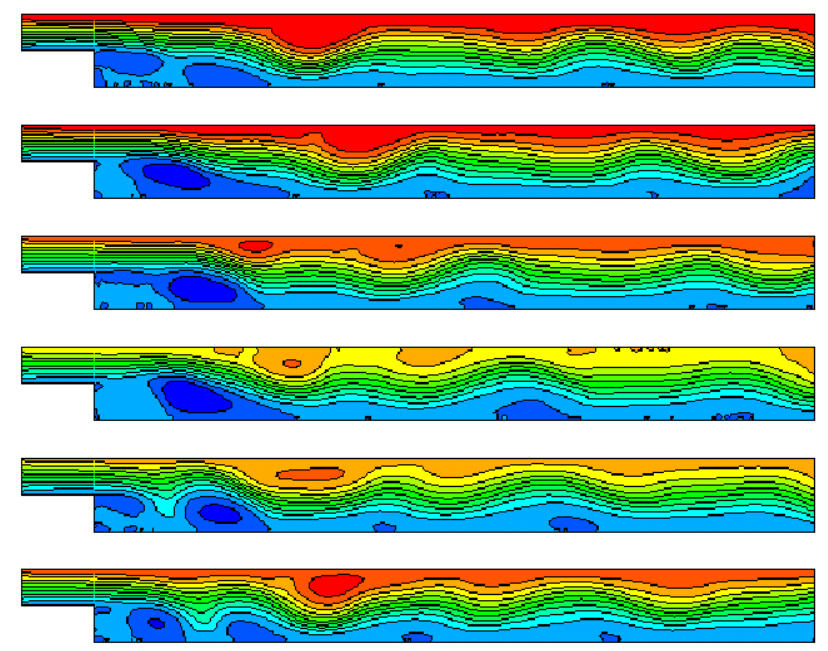

Fig. 11. Streamlines from $t=592.5$ to $t=600.0$, with $\Delta t=1.5$, closed-loop controlled (non-intrusive) regime. 


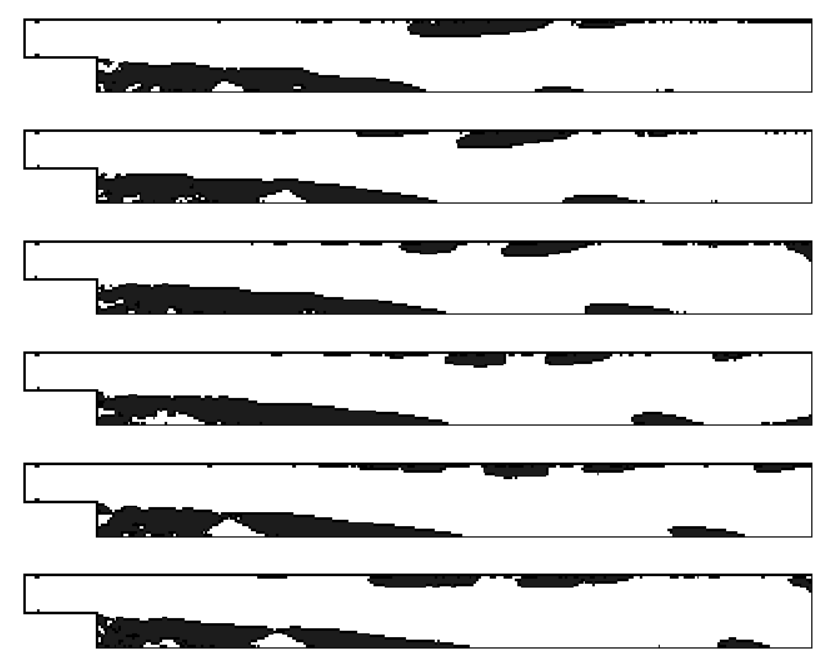

Fig. 12. Horizontal velocity $u<0$ from $t=592.5$ to $t=600.0$, with $\Delta t=1.5$, uncontrolled regime.
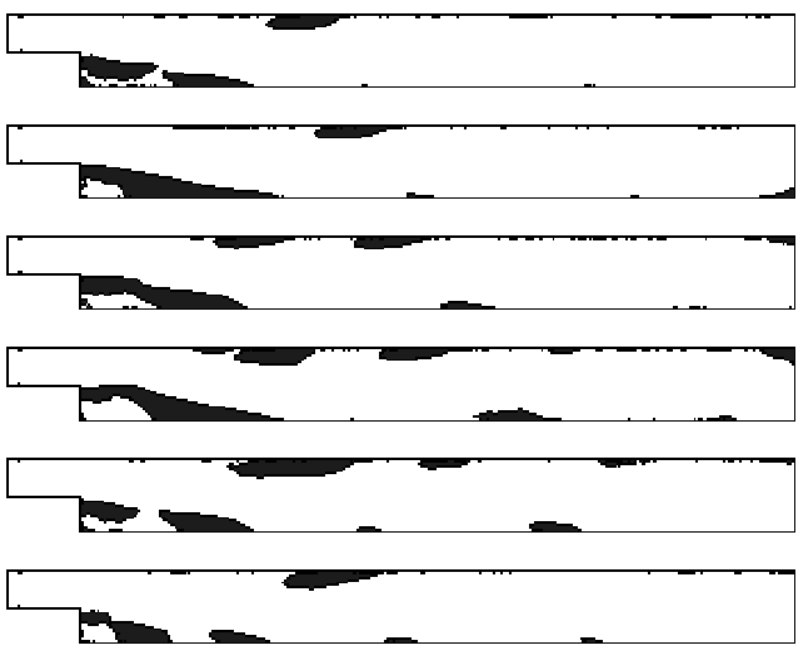

Fig. 13. Horizontal velocity $u<0$ from $t=592.5$ to $t=600.0$, with $\Delta t=1.5$, closed-loop controlled (non-intrusive) regime.

\subsubsection{Intrusive sensors}

We now wonder if it would be possible to improve results obtained with the previous non-intrusive closed-loop control. Using intrusive sensors seems to be an alternative way. Even if it is not possible to use such sensors without perturbating the flow, it can be used as an interesting step to understand and modify the flow behavior related to transport phenomena inside the channel. This control consists in the following velocity definition:

$$
u_{i n}(t)=1.0+2 A\left(\frac{u_{S_{i}}(t)-\overline{u_{S_{i}}}}{u_{S_{i}}^{\max }-u_{S_{i}}^{\min }}\right)
$$




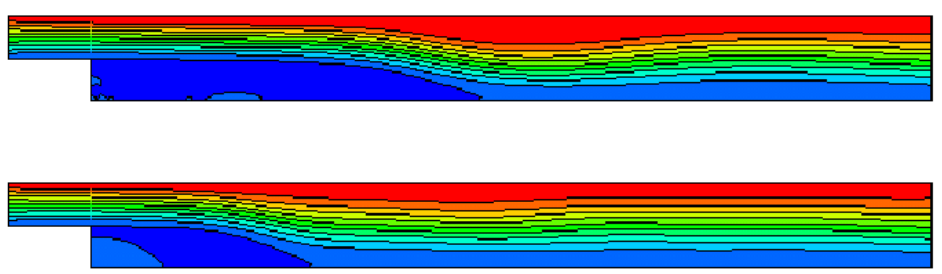

Fig. 14. Mean streamlines for uncontrolled (up) and closed-loop (non-intrusive) controlled (down) regime.

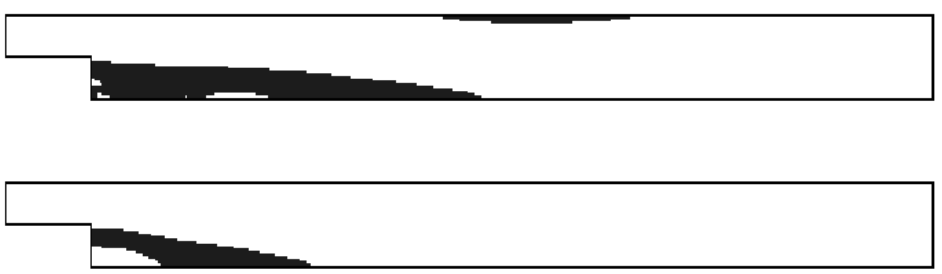

Fig. 15. Mean horizontal velocity $u<0$ for uncontrolled (up) and closed-loop (non-intrusive) controlled (down) regime.

\begin{tabular}{|l|c|c|c|c|}
\hline & Uncontrolled & Open-loop & Closed-loop non intrusive & Closed-loop intrusive \\
\hline Smallest value of $\overline{L_{r}}$ & 4.51 & 2.08 & 2.71 & 2.38 \\
\hline Smallest value of $\overline{\overline{J_{\omega}}}$ & 63.53 & 55.32 & 54.52 & 52.64 \\
\hline Smallest value of $\overline{\overline{J_{E}}}$ & 2.45 & 2.24 & 2.16 & 2.09 \\
\hline Highest value of $\overline{\overline{J_{W}}}$ & 122.09 & 185.75 & 146.13 & 159.95 \\
\hline Smallest value of $\overline{\overline{J_{F}}}$ & 3.19 & 3.13 & 3.15 & 3.15 \\
\hline
\end{tabular}

Table 6

Values of the functionals for different active control techniques.

where the intrusive sensor $S_{i}$ is located at $x=6$ and $y=0.33$, and where $\overline{u_{S_{i}}}$, $u_{S_{i}}^{\min }$ and $u_{S_{i}}^{\max }$ are respectively the average, the minimum and the maximum values of the uncontrolled velocity $u_{S_{i}}(t)$ computed at the same time and space location. Therefore, the spectrum of this signal is the same as the one recorded on the sensor $S_{i}$. Using the previous results the amplitude is set equal to $A=0.2$, leading to an inlet velocity $u_{\text {in }}$ oscillating between 0.8 and 1.2 , that has been verified by numerical tests. Values of the functionals $\overline{L_{r}}, \overline{\overline{J_{\omega}}}$, $\overline{\overline{J_{E}}}, \overline{\overline{J_{W}}}$ and $\overline{\overline{J_{F}}}$ are reported on table 6 and compared to the uncontrolled case, the best result obtained with the open-loop control, and the best result obtained with closed-loop and non intrusive sensors. Moreover, numerical tests indicated that the behavior of $\overline{J_{\omega}}$ (respectively $\overline{J_{E}}$ ) is similar to the one of $\overline{\overline{J_{\omega}}}$ (respectively $\overline{\overline{J_{E}}}$ ). They show exactly the same evolution.

As the table 6 indicates, the control effect in the intrusive case is slightly more efficient than in the non intrusive one. This behavior can be explained 
as follows: in the non-intrusive case the sensors are placed on the wall that is quite noisy because the vorticity generation procedure and local near-wall effects. It perturbates the captured frequencies that are used for the control task. This perturbation slightly affects the control efficiency. In the intrusive configuration, the sensors are placed inside the flow that is less perturbated by the wall effects, and so the flow dominating basic frequencies are better captured and then control is more efficient.

To verify this phenomenon the inlet velocity $u_{i n}(t)$ time history is plotted for both non-intrusive and intrusive cases from $t=280$ to $t=400$ (Figure 16). It should be noted that control starts at $t=300$. As the figure shows the intrusive velocity has a regular quasi-periodical evolution, but the non-intrusive one is quite perturbated and oscillating around the quasi-periodic evolution.

The intrusive control results are also very close to the best open-loop control results. It prooves that this closed-loop control approach is almost an ideal noise-free frequency capturing tool, permitting to record precisely the flow's dominant frequencies.
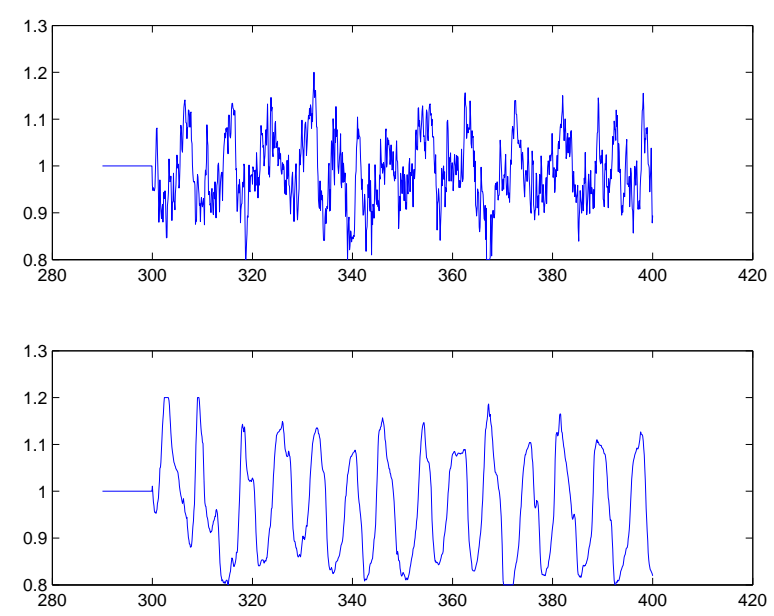

Fig. 16. Signal of $u_{i n}(t)$ for closed-loop control: with non intrusive sensor located at $x=6$ (top) and with intrusive sensor located at $x=6$ and $y=0.33$ (bottom).

\subsection{Control with active jets on the step wall}

In this section two small jets are implemented on the upper and lower parts of the vertical step (Figure 6, Control $_{2}$ ). The main target of this control technique is to influence directly the vortex shedding and the main recirculation zone: the upper jet changes the shedding dynamics and the lower one perturbates and pushes away the recirculation zone. The flow around the step is characterized by the vortex shedding process and the roll-up of the separating boundary layer into Kelvin-Helmholtz type shear layer vortices [24]. Implementing actuators on two upper and lower levels of the step modifies 
both of the above characteristics and then is a useful control tool to study. The actuators placed in the close vicinity of the bottom and the top of the step, blow or expell an horizontal flow inside the flow. On the level 2 grid, the modelisation of each actuator is performed using two points of the mesh. This geometrical configuration will remain unchanged during all the study. In following, different possibilities as steady open-loop, active open-loop and active closed-loop actuators are applied to this configuration and their efficiency is verified. All computations are performed with a level 2 grid that has been validated in section 1.3., and the final results are verified comparing to a level 3 grid. The control efficiency is measured using the average flow field and the same functionals as in the previous section. Especially, the following functionals should decrease (respectively increase) to get a good control with step jet

configuration: $\overline{L_{r}}, \overline{J_{\omega}}, \overline{J_{E}}$ (respectively $\overline{\overline{J_{W}}}$ ). The inlet velocity is taken equal to unity; $u_{j u}$ and $u_{j b}$ correspond to the uniform jet velocities respectively on the top and the bottom of the step. Then, the oscillation should be imposed to these velocities.

\subsubsection{Open-loop control}

In this subsection the efficiency of several open-loop control techniques on the flow mechanism is studied. The first range of results is obtained with steady jets on the step walls. Then, other studies with pulsing open-loop approaches are presented. In order to find the most efficient open-loop control setups, a parametric study on the choice of the frequency and the amplitude values is performed.

Control with steady jets- The simplest control actuator is a constant jet blowing or sucking a steady flow inside the main fluid. Several computations with different positive and negative velocities on the upper and lower parts of the step were performed. All of these results will not be shown here, but some important remarks are resumed as follows:

(1) To perturbate efficiently the vortex shedding and to reduce the size of the recirculation zone the suction should take place in the top of the step and the blowing in the bottom. In fact, this configuration creates a momentum, breaking the large recirculation zone and pushing it out of the diffusive formation zone.

(2) To achieve the best functionals and to break down the recirculation zone the suction should be stronger than the blowing. This setup is justified, because the suction is just behind the shedding region and a higher energy is necessary to cross it. 


\begin{tabular}{|l|c|c|c|c|c|c|c|}
\hline$\left(u_{j u}, u_{j b}\right)$ & $(0,0)$ & $(-1.0,1.0)$ & $(-0.5,0.5)$ & $(0.5,-0.5)$ & $(-1.0,0.5)$ & $(-1.0,0.25)$ & $(-0.5,0.25)$ \\
\hline \hline$\overline{L_{r}}$ & 4.5 & 3.43 & 3.97 & 3.46 & 2.31 & 1.90 & 3.35 \\
\hline$\overline{J_{\omega}}$ & 51.6 & 47.0 & 49.6 & 52.4 & 44.1 & 47.3 & 48.0 \\
\hline$\overline{\overline{J_{W}}}$ & 122 & 122 & 127 & 89 & 166 & 217 & 148 \\
\hline
\end{tabular}

Table 7

Effect of permanent jet actuators on different global functionals.

In the Table 7 the effect of different constant jet devices on three control functionals is resumed. As the Table shows the average recirculation length $\overline{L_{r}}$ is considerably reduced when the jet velocity coefficient is equivalent to $\left(u_{j u}, u_{j b}\right)=(-1.0,0.5)$ or $(-1.0,0.25)$, where $u_{j u}$ and $u_{j b}$ are respectively the upper and lower jet velocities. The mean vorticity $\overline{J_{\omega}}$ is decreased and near wall shear stresses $\overline{\overline{J_{W}}}$ related to the reduction of the large recirulation zone are increased. It confirms the above-mentioned remarks and then for the open-loop control the flow oscillates around the configuration $\left(u_{j u}, u_{j b}\right)=(-1.0,0.5)$. It should be outlined that in the oscillating cases, $\left(u_{j u}, u_{j b}\right)$ are the coefficients of the amplitude $A$ related to the upper and the lower jets.

Control with pulsing jets- In this section two open-loop approaches with step jets are studied. In the first case the actuators are pulsed by: $u_{j}(t)=$ $\left(u_{j u}, u_{j b}\right)(A+A \cos (2 \pi f t))$. It means that in both devices the jet varies between 0 and $|2 A|$, with a negative jet in the upper side of the step and a positive one in the lower part. Here, the influence of the amplitude $A$ and the frequency $f$ on the flow behavior is explored. First, for a constant frequency $f=0.2$ (close to the one used in the previous section) the amplitude is varied with $A=0.5,1.0$ and 2.0. The table 8 shows the functional values for different amplitudes compared to uncontrolled case. As the figure shows the recirculation length linearly decreases with amplitude, as well as the energy. The results for the enstrophy give an optimal value for $A=1.0$ and the wall stresses show a reasonable increase for all cases. These results show that as a compromise the amplitudes $A=0.5$ or $A=1.0$ are both efficient to control the flow with a slight energy consuming.

Otherwise, a large frequency analysis for frequencies in the gap $[0.1 ; 2.0]$ with a 0.1 stepping was performed. The amplitude was taken as $A=1.0$. The results were almsot like each other and the only observed difference was the slight proportionality of $\overline{L_{r}}$ value with the frequency. It appears that for frequencies larger than $f=0.05$ the efficiency of the control very slowly decreases increasing the frequency. This result confirms the observed behavior for the frequency in the previous section.

In the second case the actuators are pulsed by: $u_{j}(t)=\left(u_{j u}, u_{j b}\right) A \cos (2 \pi f t)$, always with $f=0.2$. It means that in both devices the jet varies between $-A$ and $A$ and there is no absolutely positive or negative device, even they never coincide to each other. For this reason we decided to perform some compu- 
Table 8

\begin{tabular}{|c|c|c|c|c|c|}
\hline & Uncontrolled & $A=0.25$ & $A=0.5$ & $A=1.0$ & $A=2.0$ \\
\hline \hline$\overline{L_{r}}$ & 4.5 & 3.46 & 2.95 & 2.04 & 1.98 \\
\hline$\overline{J_{\omega}}$ & 51.6 & 47.5 & 44.9 & 37.5 & 40.5 \\
\hline$\overline{J_{E}}$ & 2.31 & 2.20 & 2.07 & 1.86 & 1.78 \\
\hline$\overline{\overline{J_{W}}}$ & 122 & 139 & 154 & 131 & 140 \\
\hline
\end{tabular}

Effect of oscillating jet actuators with $u_{j}(t)=\left(u_{j u}, u_{j b}\right)(A+A \cos (2 \pi f t))$ on different global functionals.

\begin{tabular}{|l|c|c|c|c|c|c|}
\hline$A\left(u_{j u}, u_{j b}\right)$ & 0.0 & $0.25(-1.0,0.5)$ & $0.5(-1.0,0.5)$ & $1.0(-1.0,0.5)$ & $1.0(-1.0,1.0)$ & $1.0(1.0,0.5)$ \\
\hline \hline$\overline{L_{r}}$ & 4.5 & 3.77 & 3.22 & 2.55 & 2.61 & 2.18 \\
\hline$\overline{J_{\omega}}$ & 51.6 & 48.3 & 45.3 & 41.2 & 45.6 & 38.3 \\
\hline$\overline{J_{E}}$ & 2.31 & 2.22 & 2.16 & 2.07 & 2.20 & 2.02 \\
\hline$\overline{\overline{J_{W}}}$ & 122 & 132 & 136 & 140 & 98 & 152 \\
\hline
\end{tabular}

Table 9

Effect of oscillating jet actuators with $u_{j}(t)=\left(u_{j u}, u_{j b}\right) A \cos (2 \pi f t)$ on different global functionals.

tations with different pulsing properties. As the table 9 shows the case with $A=1.0$ and $\left(u_{j u}, u_{j b}\right)=(-1.0,0.5)$ gives a very good compromise concerning all controlled functionals. Another altenative is to use an actuator with $A=1.0$ and $\left(u_{j u}, u_{j b}\right)=(-1.0,1.0)$, where the inlet and outlet velocities are equivalent, then there is no energy add or loss in the system.

To verify several implemented techniques in this section the mean streamlines are plotted for three different controlled flows and compared to the uncontrolled case (Figure 17). As the figures show the three different control techniques reduce remarquably the recirculation area growth and stop the shedding progress.

\subsubsection{Closed-loop control}

The closed-loop control in this section is focused only on non-intrusive sensors because they are more practical in engineering applications, also because the channel flow is directly influenced by step jets a then the noise-free behavior oberved in the previous section for the intrusive sensors has less meaning here. Finally, the control with step jets is a more local technique than the inlet flow and the near-wall frequencies are enough energetic to implement this control. As the previous section the closed-loop control is used to vary the flow around $\left(u_{j u}, u_{j b}\right)=(-1.0,0.5)$. The first closed-loop control formula is similar to the 

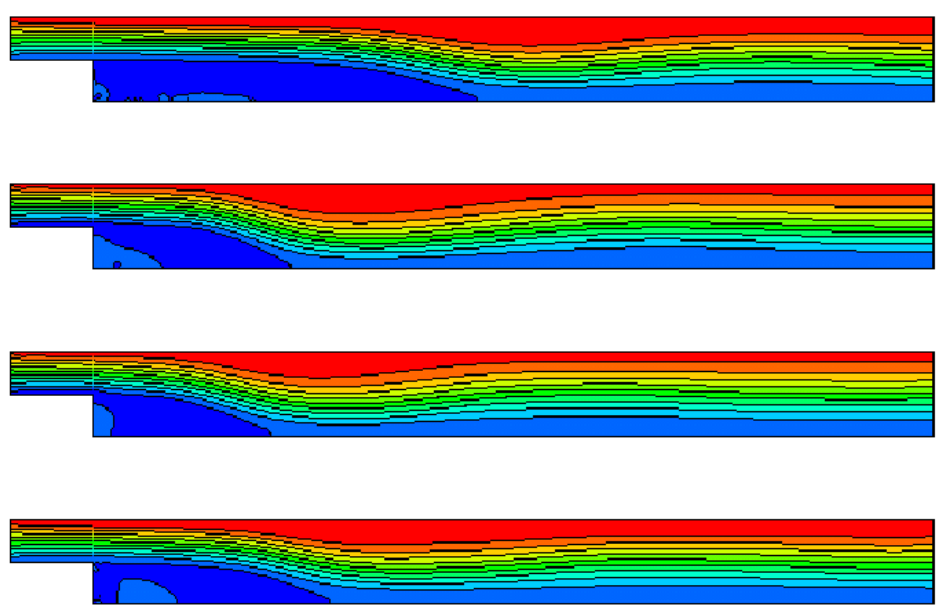

Fig. 17. Mean streamlines (from up to down) for uncontrolled, uniform jet control $\left(u_{j u}, u_{j b}\right)=(-1.0,0.5)$, open-loop with $u_{j}(t)=\left(u_{j u}, u_{j b}\right)(A+A \cos (2 \pi f t))$ and open-loop control with $u_{j}(t)=\left(u_{j u}, u_{j b}\right) A \cos (2 \pi f t)$ where $A=1.0$ and $\left(u_{j u}=-1.0, u_{j b}=0.5\right)$.

one in the section 2.2 , and is given by:

$$
u_{j}(t)=\left(u_{j u}, u_{j b}\right)\left(A+M\left(\partial^{2} u / \partial y^{2}(t)\right)_{S_{n}}\right.
$$

(referred as $C L C 1$ ). The amplitude of the negative upper jet varies between 0 and $2 A$, while the amplitude of the positive lower jet varies between 0 and $A$. It was made possible by choosing empirically the $M$ parameter. The study here consists in implementing the sensors on two locations $x=4$ and $x=7$ on top of the channel bottom wall. Also, the effect of the amplitude on the control efficiency is verified. In the table 10 the effect of the amplitude choice is verified for both sensors. As the table shows the control efficiency almost linearly increases with the amplitude value. This behavior seems natural because increasing the amplitude the energy induced in order to change the shedding and the recirculation zone is increased. Nevertheless, even for small amplitudes (e.g. $A=0.25$ ) the flow functionals are improved by this closed-loop control. Otherwise, the table shows that the sensor placed at $x=7$ is slightly more efficient than the sensor at $x=4$. It is due to the fact that at $x=4$ the flow is still influenced by the large recirculation zone behind the step. The best reults are achieved for $A=1$. at $x=7$ where the average recirculation length is reduced more than half and all other functionals also are considerably improved. In the second case the actuators are pulsed by: $u_{j}(t)=\left(u_{j u}, u_{j b}\right) M\left(\partial^{2} u / \partial y^{2}(t)\right)_{S_{n}}$ (referred as $C L C 2$ ). This definition means that the amplitude of the upper jet varies between 0 and $A$, while the amplitude of the lower jet varies between 0 and $A / 2$, but this time there is no absolutely positive or negative setup. The flow showing slightly better results using the sensor $x=7$, only the computations related to this later actuator are reported. As the table 11 indicates the results are again linearly improved increasing the amplitude value. How- 


\begin{tabular}{|l|c|c|c|c|c|c|c|}
\hline$(x, A)$ & uncontrolled & $(4,0.25)$ & $(4,0.5)$ & $(4,1.0)$ & $(7,0.25)$ & $(7,0.5)$ & $(7,1.0)$ \\
\hline \hline$\overline{L_{r}}$ & 4.5 & 3.5 & 3.1 & 2.1 & 3.4 & 2.9 & 1.9 \\
\hline$\overline{J_{\omega}}$ & 51.6 & 48.0 & 45.7 & 39.0 & 47.8 & 45.1 & 37.8 \\
\hline$\overline{J_{E}}$ & 2.31 & 2.19 & 2.12 & 1.90 & 2.18 & 2.08 & 1.86 \\
\hline$\overline{\overline{J_{W}}}$ & 122 & 140 & 149 & 152 & 139 & 155 & 151 \\
\hline
\end{tabular}

Table 10

Effect of closed-loop jet actuators on global functionals with two different sensors located at $x=4.0$ and $x=7.0$ for different jet amplitudes and negative upper and positive bottom jets $(C L C 1)$.

\begin{tabular}{|l|c|c|c|c|c|}
\hline$A\left(u_{j u}, u_{j b}\right)$ & uncontrolled & $0.25(-1,0.5)$ & $0.5(-1,0.5)$ & $1.0(-1,0.5)$ & $1.0(-1,1)$ \\
\hline \hline$\overline{L_{r}}$ & 4.5 & 3.7 & 3.2 & 2.7 & 2.9 \\
\hline$\overline{J_{\omega}}$ & 51.6 & 47.9 & 44.9 & 41.7 & 44.0 \\
\hline$\overline{J_{E}}$ & 2.31 & 2.22 & 2.16 & 2.11 & 2.15 \\
\hline$\overline{\overline{J_{W}}}$ & 122 & 130 & 134 & 135 & 123 \\
\hline
\end{tabular}

Table 11

Effect of closed-loop step jet actuators with a sensor located at $x=7.0$ and different amplitudes on global functionals and both jets oscillating around zero (CLC2).

ever, the control is less impressive than the previous case because the velocity differences between the two jets are in average smaller for this control. Furthermore, as the table shows, even for jet velocities with the same absolute value $\left(\left(u_{j u}, u_{j b}\right)=(-1.0,1.0)\right)$, where the added energy flux is almost zero, the control is still very efficient and the mean recirculation length is sensibly reduced from 4.5 to 2.9. This result underlines the main advantage of this closed-loop control approach that permits to implement an efficient zero-flux control strategy.

Finally, in figure 18 the mean streamlines for three different closed-loop control cases are compared to the uncontrolled flow. As the figure shows the CLC1 technique due to its higher flux gradients is the most efficient one and almost reduces the recirculation zone until about one third of the uncontrolled case. Nevertheless, using the $C L C 2$ method also suitable results are ahieved, even implementing a zero-flux approach. We can conclude this part with two remarks. First, not only the closed-loop control is very convenient to achieve efficient control results but also it avoids heavy trial and error calculations to choose the control modes. Second, even if the best results are obtained with significant energy supplies, a convenient control can also be achieved using lower amplitudes or nullifying the flux flowrate.

To verify the grid convergence for this family of control approachs, some of 
these controlled flows are computed using a fine level 3 grid and compared to the uncontrolled flow. The observed trends of the flow behavior, like the decrease or the increase of the functionals or the average streamlines, are always similar to the results obtained by the level 2 grid and confirm the numerical convergence.

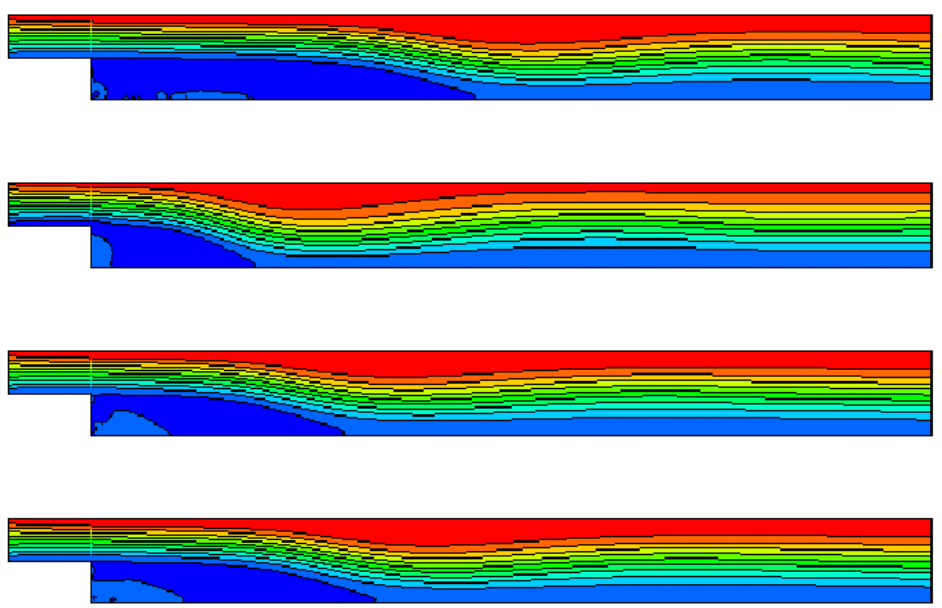

Fig. 18. Mean streamlines (from up to down) corresponding respectively to uncontrolled, $C L C 1$ with $\left(u_{j u}, u_{j b}\right)=(-1.0,0.5)$ and $A=1.0, C L C 2$ with $\left(u_{j u}, u_{j b}\right)=(-1.0,0.5)$ and $A=1.0, C L C 2$ with $\left(u_{j u}, u_{j b}\right)=(-1.0,1.0)$ and $A=1.0$ closed-loop strategies.

\section{Conclusion}

In this work, both open and closed-loop control methods were applied to control a backward-facing step flow. Computations were performed using a Vortex-In-Cell method and adapted to this control procedure. The numerical convergence was then detaily verified and compared to experimental results for transitional flows. Then, two control configurations were studied, the first configuration correponding to a pulsing inlet flow and the second one to jets introduced into the lower and upper parts of the step. The achieved results for the first strategy showed that the natural fundamental frequency of the flow offers the characteristic value to tune the pulsing frequency. The control should therefore be based on this value whatever the used devices. We also observed the efficiency of implementing the intrusive sensors. This approach improves the closed-loop control results, reducing the near-wall flow fluctuations. With an appropriate control, the flow functionals like the recirculation length, the global flow energy or the enstrophy were dramatically reduced.

For the second strategy we observed that the best control results are achieved with a high velocity negative upper jet compared to a positive bottom jet. This result was also true for oscillating jets. Then, an effcient closed-loop 
control technique was build using the above considerations. As an important conclusion, it was verified that an automatic closed-loop approach can provide a control as efficient as the best open-loop control. This observation confirms the essential importance of robust closed-loop control methods as the most practical techniques designed to manipulate today's fluid flow problems.

\section{References}

[1] Armaly, B.F., Durst, F., Pereira, J.C.F., and Schonung, B., Experimental and theoretical investigation of backward-facing step flow, J. Fluid Mech., 127, pp. 473-496 (1983).

[2] Beale, T. and Majda, A., Rates of convergence for viscous splitting of the Navier-Stokes equations, Math. Comp., 37, pp. 243-259 (1981).

[3] Chorin, A.J., Numerical study of slightly viscous flow, J. Fluid Mech., 57, pp. 785-796 (1973).

[4] Chorin, A.J., Vortex sheet approximation of boundary layers, J. Comput. Phys., 27, pp. 428-442 (1978).

[5] Christiansen, J.P., Numerical Simulation of Hydrodynamics by the Method of Point Vortices, J. Comp. Phys., 13, pp. 363-379 (1971).

[6] Cottet, G.H. and Koumoutsakos, P., Vortex methods : Theory and Practise, Cambridge University Press (2000).

[7] Creusé, E. and Mortazavi, I., Simulation of Low Reynolds Number Flow Control over a Backward-Facing Step Using Pulsed Inlet Velocities, AMRX, 4, pp. 133$152(2004)$.

[8] Creusé, E., Comparison of active control techniques over a dihedral plane : Journal of Control, Optimisation and Calculus of Variations, 6, pp. 446-466 (2001).

[9] Cruchaga, M., A study of the backward-facing step problem using a generalized streamline formulation, Commun. Numer. Meth. Engng. 14, pp. 697-708 (1998).

[10] Chun, K.B. and Sung, H.J., Control of turbulent separated flow over a backward-facing step by local forcing, Experiments in Fluids, 21, pp. 417-426 (1996).

[11] Ghoniem, A. F. and Gagnon, Y., Vortex Simulation of Laminar Recirculating Flows, J. Comp. Phys., 68, pp. 346-377 (1987).

[12] Ghoniem, A. F. and Sherman, F.S., Grid-free simulation of diffusion using random walk methods, J. Comp. Phys., 61, pp. 1-37 (1985). 
[13] Hammond, E.P., Bewley, T.R. and Moin, P., Observed mechanisms for turbulence attenuation and enhancement in opposition-controlled wall-bounded flows, Physics of Fluids, 10, 9, pp. 2421-2423 (1998).

[14] Joshi, S.S., Speyer, J.L. and Kim, J., A systems theory approach to the feedback stabilization of infinitesimal and finite-amplitude disturbances in plane Poiseuille flow, J. Fluid Mech., 332, pp. 157-184 (1997).

[15] Kim, J. and Moin, P., Application of a fractional-step method to incompressible Navier-Stokes equations, J. Comp. Phys., 59, pp. 308-323 (1985).

[16] Kaiktsis, L., Monkewitz, P., Global destabilization of flow over a backwardfacing step, Physics of fluids, 15, 12, pp. 3647-3658 (2003).

[17] Lighthill M.J.,, Boundary Layer Theory, edited by J. Rosenhead, Oxford University Press, pp. 54-61 (1963).

[18] Le, H., Moin, P. and Kim, J., Direct numerical simulation of turbulent flow over a backward-facing step, J. Fluid Mech., 330, pp. 349-374 (1997).

[19] Lee, T., Mateescu, D., Experimental and Numerical investigation of 2-D backward-facing step flow, J. Fluids and Structures, 12, pp. 703-716 (1998).

[20] Mortazavi I. and Giovannini A., The simulation of vortex dynamics downstream of a plate separator using a vortex-finite element method, International Journal of Fluid Dynamics, 5, pp. 31-48 (2001).

[21] Mortazavi, I., Micheau, P. and Giovannini A., Numerical convergence of the Random Vortex Method for complex flows, ESAIM: Proceedings, 1, pp. 521538 (1997).

[22] Mortazavi, I., Micheau, P. and Giovannini, A., Etude de la convergence numérique d'une méthode vortex pour un écoulement à grand nombre de Reynolds dans un mélangeur, Comtes Rendus Acad. Sci. Paris - Serie IIb, 330, pp. 409-416 (2002).

[23] Mouza, A. A., Pantzali, M. N., and Paras, S.V., Experimental and numerical study of backward-facing step flow, 5eme National Chemical Engineering Conference, Thessaloniki, Greece (2005).

[24] Pastoor, M., King, R., Noack, B.R., Dillman, A., and Tadmor, G., Model-Based coherent-structure control of turbulent shear flows using low-dimensional vortex models, 33 AIAA Fluids Conference and Exhibit, June 23-26, 2003, Orlando, Florida.

[25] Pellerin, S., Interactions d'une structure tourbillonnaire avec une couche limite de plaque plane. Analyse et caractrisation des phnomnes arodynamiques. $P h D$ Thesis, 1997, University Toulouse 3, France.

[26] Sethian, J.A. and Ghoniem, A.F., Validation Study of Vortex Methods, J. Comp. Phys., 74, pp. 283-317 (1988). 
[27] Spazzini, P.G., Iuso, G., Onorato, M., Zurlo, N. and Di Cicca, G.M., Unsteady behavior of back-facing step flow, Experiments in Fluids, 30, pp. 551-561 (2001).

[28] Wengle, H., Huppertz, A., Barwolff, G. and Janke, G., The manipulated transitional backward-facing step flow : an experimental and direct numerical simulation investigation, Eur. J. Mech. B- Fluids, 20, pp. 25-46 (2001).

[29] Wee, D., Yi, T., Annaswamy, A. and Ghoniem, A.F., Self-sustained oscillations and vortex shedding in backward-facing step flows : Simulation and linear instability analysis, Physics of Fluids, 16, 9, pp. 1-12 (2004).

[30] Yi, T., Annaswamy, A.M. and Ghoniem, A.F., Self-sustained oscillations in separating flows. II : Reduced-order Modeling and Control, MIT, Cambridge, MA 02139, USA (2004).

[31] Yi, T., Wee, D., Annaswamy, A.M., and Ghoniem, A.F., Self-sustained oscillations in separating flows. I : Simulation, stability and reduced-order modeling, MIT, Cambridge, MA 02139, USA (2004). 